\title{
EFEITOS DE DIFERENTES DOSAGENS DE ADUBAÇÃO NO CRESCIMENTO INICIAL DE Eucalyptus benthamii Maiden et Cambage
}

\author{
EFFECTS OF DIFFERENT FERTILIZER DOSAGES ON THE INITIAL GROWTH OF Eucalyptus \\ benthamii Maiden et Cambage
}

\author{
Edson Luis Serpe ${ }^{1}$, Antonio Carlos Vargas Motta ${ }^{2}$, Afonso Figueiredo Filho ${ }^{3}$, Julio Eduardo Arce ${ }^{4}$ \\ ${ }^{1}$ LWARCEL, Lençóis Paulista, São Paulo, Brasil-serpe.edson@gmail.com \\ 2,4 Universidade Federal do Paraná, Curitiba, Paraná, Brasil -mottaufpr@gmail.com \& jarce@ufpr.br \\ ${ }^{3}$ Universidade Estadual do Centro Oeste, Irati, Paraná, Brasil - afigfilho@gmail.com
}

\section{RESUMO}

Os efeitos de diferentes dosagens de adubação no crescimento inicial de Eucalyptus benthamii Maiden et Cambage foram avaliados em General Carneiro, região sul do Paraná. O objetivo da pesquisa foi avaliar o crescimento e a produção em volume total, comercial e por sortimento em região com invernos rigorosos. Foi utilizado um delineamento inteiramente casualizado com amostras de $250 \mathrm{~m}^{2}$ (seis tratamentos e oito repetições). Os dados de diâmetros e alturas, foram utilizados para o ajuste de equações hipsométricas e funções de afilamento. Os resultados obtidos, foram submetidos ao teste de Bartlett, análise de variância (ANOVA) e o teste de Tukey. Evidencia-se que a aplicação de adubação de base e adubações de cobertura em diferentes quantidades, formulações e períodos de aplicação, proporcionam distintos efeitos no crescimento em volume comercial e total. Observou-se que espécie é resistente as geadas frequentes no sul do Brasil, atingindo incremento médio anual de até $54,4 \mathrm{~m}^{3}$.ha-1. ano $^{-1}$.

PALAVRAS-CHAVE: Geadas, Modelagem, Nutrição Florestal, Preparo de solo, Produção.

\section{ABSTRACT}

The effects of different dosages of fertilization on the initial growth of Eucalyptus benthamii Maiden et Cambage were evaluated in General Carneiro, southern region of Paraná. The objective of the research was to evaluate the growth and production in total volume, commercial and by assortment in region with rigorous winters. A completely randomized design with $250 \mathrm{~m}^{2}$ samples (six treatments and eight replicates) was used. The data of diameters and heights were used for the adjustment of hipsometric equations and taper functions. The results were submitted to the Bartlett test, analysis of variance (ANOVA) and the Tukey test. It has been shown that the application of base fertilization and cover fertilizations in different quantities, formulations and periods of application, provide different effects on growth in commercial and total volume. It was observed that the species is resistant to frequent frosts in southern Brazil, reaching an annual average increase of up to $54.4 \mathrm{~m}^{3} \cdot$ ha $^{-1}$.year-1.

KEYWORDS: Frosts, Modeling, Forest Nutrition, Soil preparation, Production. 


\section{INTRODUÇÃO}

As plantações florestais no Brasil em 2016, totalizaram 7,84 milhões de hectares (0,9\% do território Nacional), sendo 5,67 milhões com eucalipto (72,3\%), 1,58 milhão com Pinus (20,2\%) e outras espécies com 0,59 milhões (7,5\%) (IBÁ, 2017). O Paraná é o estado com maior área plantada de Pinus (663.600 ha), mas gradativamente está apresentando migração na área plantada para o eucalipto (283.500 ha) (IBÁ, 2017).

A definição da espécie a ser plantada está relacionada com o uso da madeira e as condições climáticas da região (ABRAF, 2013). As espécies de eucalipto economicamente importantes para as condições mais frias do Brasil constituem-se um grupo muito restrito: Eucalyptus benthamii Maiden et Cambage, Eucalyptus dunnii Maiden, Eucalyptus saligna Smith, Eucalyptus grandis Hill ex Maiden, e o híbrido entre $E$. benthamii $x$ E. dunnii (PALUDZYSZYN FILHO et al., 2006).

O $E$. benthamii é uma excelente opção para reflorestamentos em regiões de clima frio, principalmente em localidades onde ocorre geadas frequentes e severas, como no sul do Brasil (GRAÇA et al., 1999). A temperatura mínima absoluta que o $E$. benthamii suporta, registrada em abrigo meteorológico é de até $-6{ }^{\circ} \mathrm{C}$ (DARROW, 1995), suportando até 25 geadas anuais (FAO, 1981).

A necessidade de adubação em espécies florestais, provém de que nem sempre o solo é capaz de fornecer todos os nutrientes em quantidade que as plantas necessitam para um adequado crescimento. A correta nutrição em plantios de eucalipto é essencial para o estabelecimento de florestas de alta produtividade (GONÇALVES, 1995).

O eucalipto responde à adubação (NPK) e, em alguns casos, é necessária a adubação com $B$ e Zn, sendo que para a maioria dos solos brasileiros a adubação com fósforo é essencial (MORA \& GARCIA, 2000). A adubação no plantio visa principalmente o fornecimento de $\mathrm{P}, \mathrm{Cu}$ e $\mathrm{Zn}$. Solos com baixo teor de matéria orgânica e de potássio deve-se também utilizar doses de $\mathrm{N}$ e K. A adubação de cobertura visa fornecer os nutrientes de alta mobilidade no solo, N, P e B (SILVEIRA et al., 2001).

$O$ fato do $E$. benthamii apresentar grande resistência a geadas, despertou o interesse na realização dessa pesquisa, a qual busca gerar conhecimentos sobre o efeito de diferentes dosagens de adubos no crescimento dessa espécie florestal para o sul do Brasil.

Assim, o objetivo da pesquisa foi avaliar o crescimento e a produção em volume total, comercial e por sortimento de $E$. benthamii em região com invernos rigorosos, sob diferentes dosagens de adubação.

\section{MATERIAL E MÉTODOS}

\section{Localização da área de estudo}

A área de estudo localiza-se no município de General Carneiro, região Centro-Sul do estado do Paraná, nas coordenadas geográficas 26018'40" Latitude Sul e 51으늘 'Longitude Oeste, em uma altitude média de 1.072 m.s.n.m.

De acordo com Köppen, o clima da região é caracterizado como clima temperado (Cfb). A estação meteorológica, localizada a $2,5 \mathrm{~km}$ da área de estudo, apresenta histórico de precipitação média anual de 1776 $\mathrm{mm}$ e temperatura média de $16,4{ }^{\circ} \mathrm{C}$ (máxima 22,9 e mínima $\left.11,7^{\circ} \mathrm{C}\right)$.

A região apresenta frequentes geadas entre os meses de maio e setembro, as quais são classificadas de acordo com a classificação de geadas para o estado de Santa Catarina (Tabela 1).

Tabela 1. Classificação de geadas

\begin{tabular}{cc}
\hline Temperatura mínima $\left({ }^{\circ} \mathrm{C}\right)$ & Fenômeno \\
\hline$>0$ & Sem Ocorrência \\
-2 a 0 & Geada Fraca \\
-4 a -2 & Geada Moderada \\
-6 a -4 & Geada Mediana \\
-8 a -6 & Geada Forte \\
$<-8$ & Geada Muito Forte \\
\hline
\end{tabular}

Fonte: Adaptado de ARAUJO et al. (2009).

A área de estudo, durante os anos de 2008 a 2013 foi atingida por 41 geadas fracas, 18 moderadas, três medianas e uma forte, conforme Tabela 2 .

Tabela 2. Distribuição das geadas em General Carneiro, PR

\begin{tabular}{cccccc}
\hline \multirow{2}{*}{ Ano } & \multicolumn{5}{c}{ Número de geadas } \\
\cline { 2 - 6 } & Fraca & Moderada & Mediana & Forte & Total \\
\hline 2008 & 6 & 3 & 2 & 0 & 11 \\
\hline 2009 & 6 & 6 & 0 & 0 & 12 \\
2010 & 4 & 1 & 1 & 0 & 6 \\
\hline 2011 & 8 & 6 & 0 & 1 & 15 \\
2012 & 7 & 1 & 0 & 0 & 8 \\
\hline 2013 & 10 & 1 & 0 & 0 & 11 \\
\hline Total & $\mathbf{4 1}$ & $\mathbf{1 8}$ & $\mathbf{3}$ & $\mathbf{1}$ & $\mathbf{6 3}$ \\
\hline
\end{tabular}

A Figura 1 mostra, os povoamentos de $E$. benthamii e $E$. dunnii atingidos por geada forte $\left(-6,4{ }^{\circ} \mathrm{C}\right)$ ocorrida em julho de 2011. O E. dunnii sofreu queima foliar, já o E. benthamii 
não apresentou tal fato.

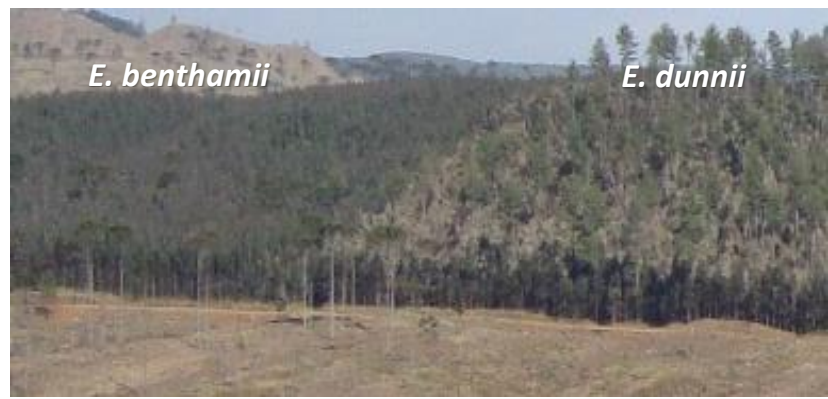

Figura 1. Área de E. benthamii e E. dunnii atingida por geada forte em julho de 2011.

Além, das ocorrências de geadas, em julho de 2013 o delineamento de $E$. benthamii, foi atingido por neve $(-0,1$ ${ }^{\circ} \mathrm{C}$ ) (Figura 2).

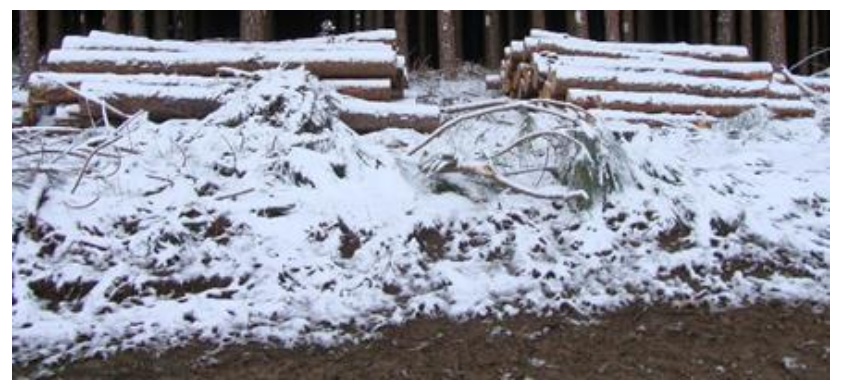

Figura 2. Ocorrência de neve em julho de 2013.

\section{Características da área de estudo}

As mudas utilizadas no experimento foram produzidas com sementes de procedência de Pomar de Sementes Clonal da localidade de Kowen na Austrália.

Em novembro de 2007 foi implantado em área com relevo plano, um delineamento inteiramente casualizado com E. benthamii sob diferentes dosagens de adubação, com unidades amostrais (repetição) de $250 \mathrm{~m}^{2}$ (seis tratamentos e oito repetições) (Tabela 3).

Tabela 3. Delineamento inteiramente casualizado

\begin{tabular}{ccccccc}
\hline Aplicação (dias) & $\mathbf{0}$ & $\mathbf{3 0}$ & $\mathbf{4 0}$ & $\mathbf{6 0}$ & $\mathbf{9 0}$ \\
\hline & & \multicolumn{6}{c}{ Adubo - NPK } \\
\cline { 2 - 7 } Tratamento & SSP* & $\mathbf{0 6}$ & $\mathbf{1 5}$ & $\mathbf{0 6}$ & $\mathbf{1 5}$ & $\mathbf{1 5}$ \\
& & $\mathbf{3 0}$ & $\mathbf{0 5}$ & $\mathbf{3 0}$ & $\mathbf{0 5}$ & $\mathbf{0 5}$ \\
& & $\mathbf{0 6}$ & $\mathbf{3 0}$ & $\mathbf{0 6}$ & $\mathbf{3 0}$ & $\mathbf{3 0}$ \\
\hline 1 & 0 & 0 & 0 & 0 & 0 & 0 \\
\hline 2 & 100 & 0 & 0 & 0 & 0 & 0 \\
3 & 100 & 75 & 0 & 75 & 0 & 75 \\
4 & 100 & 150 & 150 & 0 & 0 & 0 \\
5 & 100 & 150 & 0 & 0 & 150 & 0 \\
6 & 100 & 150 & 0 & 0 & 150 & 150 \\
\hline
\end{tabular}

SSP: Superfosfato simples; N: Nitrogênio; P: Fósforo; K: Potássio; *: g. planta $^{-1}$.
A adubação utilizada além de conter macronutrientes contém micronutrientes em sua formulação. $O$ adubo NPK 06-30-06 possui os micronutrientes $B(0,05 \%)$ e $\mathrm{Zn}(0,2 \%)$, já o adubo NPK $15-05-30$ possui B $(0,2 \%)$ e Zn (0,02\%). Na Tabela 4 está detalhada a quantidade de adubação total aplicada por hectare.

Tabela 1. Quantidade de adubação aplicada por hectare

\begin{tabular}{cccccccc}
\hline Tratamento & $\mathbf{N}$ & $\mathrm{P}_{2} \mathrm{O}_{5}$ & $\mathbf{K}_{2} \mathbf{O}$ & $\mathbf{C a}$ & $\mathbf{S}$ & $\mathbf{B}$ & $\mathbf{Z n}$ \\
\hline $\mathbf{1}$ & 50,4 & 112,8 & 86,4 & 30,4 & 17,6 & 0,6 & 0,5 \\
$\mathbf{2}$ & 50,4 & 112,8 & 86,4 & 30,4 & 17,6 & 0,6 & 0,5 \\
$\mathbf{3}$ & 32,4 & 106,8 & 50,4 & 30,4 & 17,6 & 0,4 & 0,5 \\
$\mathbf{4}$ & 86,4 & 124,8 & 158,4 & 30,4 & 17,6 & 1,1 & 0,6 \\
$\mathbf{5}$ & 0,0 & 28,8 & 0,0 & 30,4 & 17,6 & 0,0 & 0,0 \\
$\mathbf{6}$ & 0,0 & 0,0 & 0,0 & 0,0 & 0,0 & 0,0 & 0,0 \\
\hline
\end{tabular}

Para o preparo de solo, foi realizada a subsolagem com $40 \mathrm{~cm}$ de profundidade, não sendo realizada a calagem. $O$ plantio foi implantado manualmente com o auxílio de pá chilena, utilizando espaçamento de $2,5 \mathrm{~m} \times 2,5 \mathrm{~m}$, e definido cada tratamento com área total de $2.000 \mathrm{~m}^{2}$ (320 árvores).

A adubação de base (plantio) foi realizada na cova no momento do plantio e as adubações de cobertura (pósplantio) por meio de covetas laterais.

\section{Coleta dos dados}

A coleta dos dados foi realizada em novembro de 2013, estando o plantio com seis anos de idade. Foram medidos todos os diâmetros a 1,3 m do solo (DAP) com fita métrica, as alturas totais de $20 \%$ das árvores com o hipsômetro Vertex, além das alturas das três árvores dominantes.

\section{Ajuste de equações hipsométricas e funções de afilamento}

Como não foram medidas todas as alturas, foram ajustados e testados seis modelos hipsométricos: Trorey (1932), Prodan (1968), Henriksen (1950), Curtis (1967), Soares et al. (2006) e Stoffels \& Van Soest (1953).

Para os ajustes das funções de afilamento visando estimar os volumes totais e comerciais (diâmetro de até 8 $\mathrm{cm}$ com casca) foram cubadas 27 árvores.

Foram ajustados e testados os modelos de afilamento de Kozak et al. (1969) modificado, o polinômio de 5으 grau ou de Schöepfer (1966) e o polinômio de potências múltiplas e fracionárias ou Modelo de Hradetzky (1976).

A seleção dos modelos hipsométricos e de afilamento mais apropriados, foi realizada considerando as estatísticas 
de erro padrão de estimativa absoluto $(m)$ e relativo e o coeficiente de determinação ajustado, além da análise gráfica dos resíduos.

Muito embora, o povoamento seja relativamente jovem, o sortimento foi também estimado por tratamento, definindo-o de acordo com o mercado de madeira regional, considerando as seguintes classes de diâmetro na ponta fina das toras e respectivos comprimentos: 8 - 17,9 $\mathrm{cm}(2,30 \mathrm{~m}), 18-24,9 \mathrm{~cm}$ e maior que $25 \mathrm{~cm}(2,65 \mathrm{~m})$.

\section{Análises estatísticas do experimento}

Para verificar os efeitos da adubação nos tratamentos, foi aplicado o teste de Bartlett para as variáveis: diâmetro, altura total, volume comercial $(\geq 8 \mathrm{~cm}$ na ponta fina da tora) e volume total.

Após verificado que as variâncias dos tratamentos são homogêneas foi realizada a análise de variância (ANOVA). Finalmente, se o teste $\mathrm{F}$ de ANOVA rejeitar a hipótese de nulidade o teste de Tukey, foi aplicado a fim de verificar as diferenças entre as médias dos tratamentos. Todos os testes consideraram um $p$-valor $\alpha \leq 0,05$.

\section{RESULTADOS E DISCUSSÃO}

Ao analisar o erro padrão de estimativa absoluto $(m)$ e relativo, o coeficiente de determinação ajustado e gráfico dos resíduos, definiu-se pelo uso dos modelos hipsométrico de Trorey (1932) e de afilamento de Hradetzky (1976).

Estes modelos apresentaram, as melhores estatísticas de ajuste e precisão, sendo utilizados os coeficientes da Tabela 5, para as estimativas de altura total.

Tabela 5. Coeficientes da equação hipsométrica

\begin{tabular}{cccc}
\hline Equação & Tratamento & $\boldsymbol{\beta}_{\mathbf{1}}$ & $\boldsymbol{\beta}_{\mathbf{2}}$ \\
\hline & 1 & 1,97939 & $-0,03831$ \\
$\mathrm{~h}=\beta_{1}$ DAP $+\beta_{2}$ DAP $^{2}$ & 2 & 1,98438 & $-0,03968$ \\
& 3 & 2,07106 & $-0,04335$ \\
& 5 & 2,00453 & $-0,04024$ \\
& 6 & 2,18949 & $-0,05060$ \\
\hline
\end{tabular}

h: Altura (m); DAP: Diâmetro a 1,3 m (cm); $\beta$ : Coeficiente de regressão.

Os coeficientes para a estimativa de diâmetros ao longo do tronco, são apresentados na Tabela 6. Estes foram utilizados na integral da função de afilamento. A equação de volume gerada pela integral possibilitou estimar o volume total, comercial e por sortimento.
Tabela 6. Coeficientes para estimativa de diâmetros ao longo do tronco

\begin{tabular}{cccc}
\hline Modelo & \multicolumn{3}{c}{ Coeficientes } \\
\hline & $\beta_{0}$ & Constante & 18,85560 \\
Hradetzky & $\beta_{1}$ & $(\mathrm{hi} / \mathrm{h})^{0,005}$ & $-18,08860$ \\
$(1976)$ & $\beta_{2}$ & $(\mathrm{hi} / \mathrm{h})^{2}$ & $-0,66465$ \\
& $\beta_{4}$ & $(\mathrm{hi} / \mathrm{h})^{10}$ & $-0,10147$ \\
\hline
\end{tabular}

$\beta$ : Coeficiente; hi: Altura da seção (m); h: Altura (m).

\section{Comparativo entre as variáveis estudadas}

O teste de Bartlett indicou que as variâncias para diâmetro, altura total, volume comercial e total são homogêneas para $\alpha \leq 0,05$.

$\mathrm{O}$ teste $\mathrm{F}$ da análise de variância (ANOVA) rejeitou a hipótese de nulidade para os tratamentos, indicando que a média de pelo menos dois tratamentos são diferentes. Assim, foi aplicado o teste de Tukey $(\alpha \leq 0,05)$ para verificar as diferenças entre as médias dos tratamentos (Tabela 7).

Tabela 7. Teste de Tukey para as variáveis estudadas

\begin{tabular}{ccccc}
\hline Tratamento & $\begin{array}{c}\text { DAP } \\
(\mathbf{c m})\end{array}$ & $\begin{array}{c}\text { Altura } \\
(\mathbf{m})\end{array}$ & $\begin{array}{c}\text { Volume } \\
\text { comercial } \\
\left(\mathbf{m}^{\mathbf{3}} \cdot \mathbf{h a}^{-\mathbf{1}}\right)\end{array}$ & $\begin{array}{c}\text { Volume } \\
\text { total } \\
\left(\mathbf{m}^{\mathbf{3}} \cdot \mathbf{h a}^{-1}\right)\end{array}$ \\
\hline $\mathbf{1}$ & $11,6^{\mathrm{c}}$ & $17,0^{\mathrm{b}}$ & $147,1^{\mathrm{c}}$ & $180,2^{\mathrm{c}}$ \\
\hline $\mathbf{2}$ & $12,6^{\mathrm{bc}}$ & $18,2^{\mathrm{ab}}$ & $169,7^{\mathrm{bc}}$ & $208,1^{\mathrm{bc}}$ \\
\hline $\mathbf{3}$ & $14,8^{\mathrm{a}}$ & $20,0^{\mathrm{a}}$ & $240,2^{\mathrm{a}}$ & $292,2^{\mathrm{a}}$ \\
\hline $\mathbf{4}$ & $13,9^{\mathrm{ab}}$ & $18,6^{\mathrm{ab}}$ & $217,0^{\mathrm{ab}}$ & $263,5^{\mathrm{ab}}$ \\
\hline $\mathbf{5}$ & $14,7^{\mathrm{a}}$ & $19,6^{\mathrm{a}}$ & $253,1^{\mathrm{a}}$ & $298,8^{\mathrm{a}}$ \\
\hline
\end{tabular}

Médias seguidas da mesma letra não diferem estatisticamente pelo teste de Tukey ao nível de $5 \%$ de significância.

Os resultados mostrados na Tabela 7 para DAP, altura, volume comercial e total, indicam efeitos da adubação sobre todos os parâmetros.

O uso isolado de supersimples, não foi suficiente para elevar de modo significativo nenhum dos parâmetros. Tal fato, pode estar associado a dose aplicada, ao solo utilizado e existência de outros nutrientes esteja limitando a produtividade.

$\mathrm{O}$ acréscimo na dose de $\mathrm{P}$ e aplicação de $\mathrm{N}$ e $\mathrm{K}$ resultaram em aumento no DAP, volume comercial e total para os tratamentos 3, 5 e 6. Tal fato era esperado uma vez que o nível de $\mathrm{K}$ no solo era baixo, possibilitando a resposta ao uso de K. É sabido também que os teores totais de $\mathrm{K}$ contido nos minerais primários, em solo de basalto são baixos e relação a solo originado de granito por exemplo e que possam atuar com reservatório de liberação lenta.

Diferentes dos três parâmetros apresentado 
anteriormente, a altura só houve diferença entre testemunha e adubado, mostrando ser menos dependente da adubação. Logo, na altura mostrou menos efetivo para avaliação da adubação na condição estudada.

Maior crescimento foi observado para tratamento 6, sem diferença estatística, sugere que aplicação de maiores doses de $\mathrm{K}, \mathrm{B}$ e $\mathrm{N}$ podem elevar a produtividade e merece ser pesquisada, de forma a determinar qual elemento de modo isolado é responsável pelo aumento.

Ainda, o incremento da dose de $\mathrm{P}$, ligeiramente acima da dose recomendada de $90 \mathrm{~kg}$.ha-1 ${ }^{-1}$ de $\mathrm{P}_{2} \mathrm{O}_{5}$, possivelmente ampliaria a possibilidade de resposta em solo com elevada capacidade de fixação, como indicado anteriormente.

Ao comparar os tratamentos T4 e T5 que receberam igual adubação, mas em diferentes períodos, identificou-se que a aplicação em doses com períodos intercalados de 30 dias, apresentam melhores resultados que a aplicação com períodos de 10 dias.

Estudos de resposta ao uso de fertilizantes no $E$. benthamii em clima similar são escassos e difícil de comparação dado a variação nas doses utilizadas.

Corroborando com resultados obtidos, Dias et al. (2014) avaliaram o uso de adubação de base e adubação de cobertura distribuída em duas aplicações aos cinco e 10 meses de idade para o cultivo de E. benthamii. Esses autores constataram resposta significativa ao primeiro ano na variável diâmetro à altura do colo. Na testemunha, o diâmetro de colo médio foi de $3,3 \mathrm{~cm}$, enquanto, nos tratamentos adubados situaram-se entre 4,9 e 5,9 cm.

Benin et al. (2014), em estudos do desenvolvimento inicial de $E$. benthamii, obtiveram ao terceiro ano o incremento médio anual em DAP de $2,9 \mathrm{~cm}$.

No presente estudo o crescimento anual em diâmetro é de 2,6 cm.ano ${ }^{-1}$ no T6 e no T1 (testemunha) de 1,9 $\mathrm{cm}$.ano-1. Quando comparado à média de crescimento em diâmetro dos trabalhos citados verificou-se que apesar do T6 ser atingido por geadas (63 ocorrências) apresentou além da resistência a geadas um crescimento diamétrico dentro da média encontrada na literatura.

A média de crescimento em altura de 3,20 m obtido no presente trabalho, foram maiores que obtidos por Stermer et al. (2007) e Dias et al. (2014). O primeiro autor constatou altura média de $3,0 \mathrm{~m}$, ao estudar o efeito da compactação e nutrição de $E$. badjensis. Já, Dias et al. (2014) constatou altura entre 1,8 e 2,9 m no primeiro ano em função da adubação.

A adubação ao aumentar o crescimento também influenciou positivamente no sortimento da tora (Figura 3).

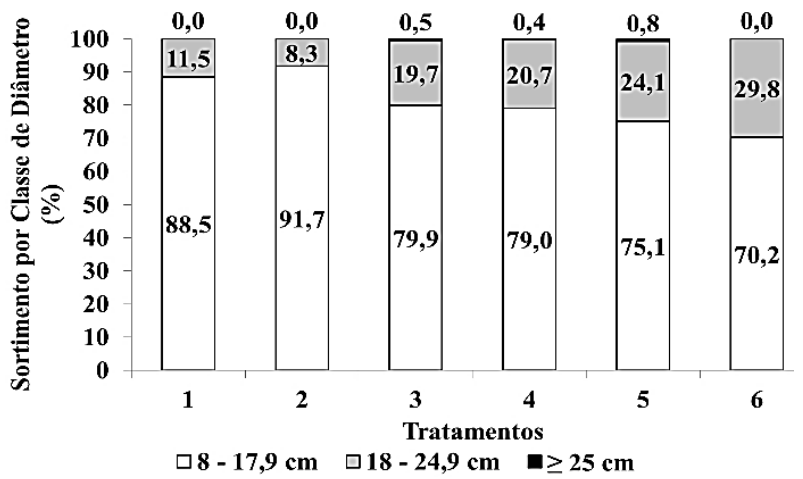

Figura 3. Sortimento por classe de diâmetro.

Os resultados do percentual de volume por sortimento em diferentes classes indicam que os tratamentos T4, T5 e T6 apresentam volume acima de $20 \%$ no sortimento maior que $18 \mathrm{~cm}$.

Quando comparado o tratamento que recebeu mais adubo (T6) com o tratamento que recebeu apenas $P$ (T2) foi verificado incremento de 8,3 para de $29,8 \%$ no volume na classe acima de $18 \mathrm{~cm}$.

O incremento médio anual (IMA) para o volume total de cada tratamento, está ilustrado na Figura 4.

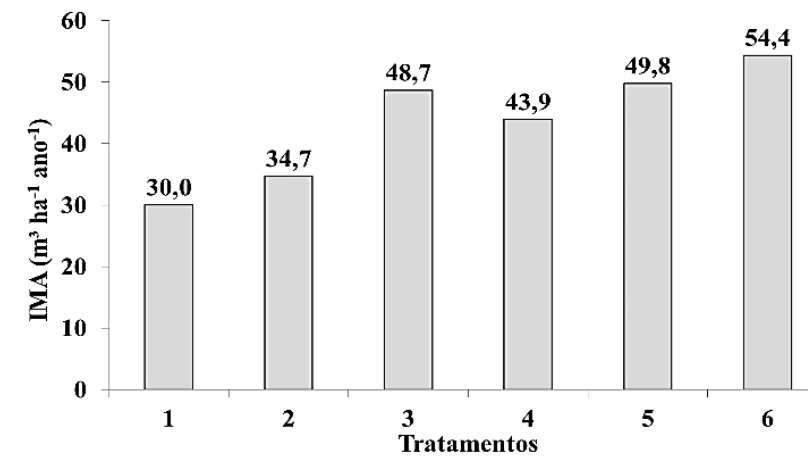

Figura 4. Incremento volumétrico médio anual.

O IMA de 30,0 $\mathrm{m}^{3}$.ha-1.ano-1 na testemunha, pode ser considerado baixo comparado a média nacional que é de $35,7 \mathrm{~m}^{3}$.ha ${ }^{-1}$.ano-1 segundo IBÁ (2017). Mas, considerando que a testemunha não recebeu fertilização e tolerou condições climáticas adversas (geadas), tal incremento não deve ser inferiorizado.

$\mathrm{O}$ valor médio do IMA dos tratamentos T3, T4, T5 e T6 é de $49,2 \mathrm{~m}^{3}$.ha ${ }^{-1}$.ano ${ }^{-1}$, onde a diferença é de $64 \%$ entre o tratamento que não recebeu adubação (T1).

A diferença é ainda mais expressiva com comparação entre testemunha $\left(30,0 \mathrm{~m}^{3} \cdot \mathrm{ha}^{-1}\right.$.ano-1 $)$ e o IMA médio anual de $54,4 \mathrm{~m}^{3}$.ha ${ }^{-1}$.ano-1 para o tratamento que recebeu mais adubo T6, o que representa $81,0 \%$ de diferença.

Gatto et al. (2003), em estudo do efeito do método de preparo do solo, em área de reforma de $E$. grandis no município de Santa Bárbara, estado de Minas Gerais, 
verificaram após 3 anos o IMA de 42,7 $\mathrm{m}^{3}$.ha-1.ano-1), enquanto para o tratamento testemunha (sem adubação) a produção total alcançada foi $\left(20,0 \mathrm{~m}^{3} \cdot \mathrm{ha}^{-1} \cdot \mathrm{ano}^{-1}\right)$.

\section{CONCLUSÕES}

A produtividade obtida sem utilização de adubação, mesmo estando abaixo da média nacional, pode ser considerada adequada, visto as inúmeras ocorrências de geadas.

O uso isolado de reduzida dosagem de fósforo, não foi suficiente para aumentar a produtividade do eucalipto.

A dosagem e aplicação de fertilizantes em períodos adequados, demonstram ser um importante investimento proporcionando elevado aumento na produtividade.

O E. benthamii demonstrou ser uma excelente opção para áreas temperadas, outrora nicho predominante do pinus.

\section{AGRADECIMENTOS}

À Remasa Reflorestadora S.A., pela autorização e apoio no trabalho de campo.

\section{REFERÊNCIAS}

ARAUJO, C.E.S. et al. Previsão de geadas em Santa Catarina. 2009. Disponível em: http://www.ciram.com.br/finep14/ recursos/Rel_Finep14_Geada.pdf

ABRAF. Anuário estatístico ABRAF 2013 ano base 2012. 2013. Disponivel em: http://www.ipef.br/estatisticas/relatorios/ anuario-abraf13-br.pdf

BENIN, C.C. et al. Initial assessments on the plantation of Eucalyptus benthamii Maiden et Cambage deployed in different spacing. Applied Research \& Agrotechnology, v.7, n.1, p.55-61, 2014.

DARROW, W.K. Selection of eucalypt species for cold and dry areas in South Africa. 1995. Disponível em: http://www.forestry.crc.org.au/iufro95.htm\#theme2

DIAS, L.P.R. et. al. Substituição parcial de fosfato solúvel por natural na implantação de Eucalyptus benthamii e Eucalyptus dunnii no Planalto Sul Catarinense. Revista Brasileira de Ciência do Solo, v.38, n.2, p.516-523, 2014.

FAO. El eucalipto en la repoblación forestal. 1981. Disponível em: http://www.fao.org/3/a-ac459s.pdf

GATTO, A. et al. Efeito do método de preparo do solo, em área de reforma, nas suas características, na composição mineral e na produtividade de plantações de Eucalyptus grandis. Árvore, v.27, n.5, p.635-646, 2003.

GONÇALVES, J.L.M. Recomendações de adubação para
Eucalyptus, Pinus e espécies típicas da Mata Atlântica. Documentos Florestais, n.15, p.1-23, 1995.

GRAÇA, M.E.C. et. al. Capacidade de rebrota e de enraizamento de Eucalyptus benthamii. Boletim de Pesquisa Florestal, n.39, p.135-138, 1999.

IBÁ. Relatório 2017. 2017. Disponível em <http://iba.org/images/shared/Biblioteca/IBA_RelatorioAnual20 16_pdf

MORA, A.L.; GARCIA, C.H. A cultura do eucalipto no Brasil. São Paulo: SBS, 2000.

PALUDZYSZYN FILHO, E. et al. Eucaliptos indicados para plantio no estado do Paraná. Documentos, n.129, p.1-45, 2006.

SILVEIRA, R.L. et. al. Seja doutor do seu eucalipto. Informações agronômicas, n.93, p.1-31, 2001.

SOARES, C.P.B. et. al. Dendrometria e inventário florestal. Viçosa: UFV, 2006.

STERMER, R.P. et al. Influência da compactação no crescimento e na nutrição de Eucalyptus badjensis. Comunicado Técnico, n.196, p.1-5, 2007. 